\title{
Hypertrabeculated left ventricle in mitochondriopathy
}

\author{
J Finsterer, C Stöllberger
}

Ludwig Boltzmann Institute for Research in Epilepsy and

Neuromuscular Disorders, Vienna, Austria

J Finsterer

Medical Department, Krankenanstalt Rudolfstiftung, Vienna, Austria

C Stöllberger

Correspondence to: Dr J Finsterer, Postfach 348, 1180 Wien, Austria.

Accepted for publication 10 June 1998
Abnormal left ventricular trabeculations can be a cardiac manifestation of Becker's muscular dystrophy. ${ }^{1}$ Abnormal left ventricular trabeculations in mitochondrial myopathy have not been reported before.

A 68 year old man with a history of chronic congestive heart failure and hypertension, had recurrent raised creatine kinase (CK) concentrations (up to $150 \mathrm{U} / 1$; normal $70 \mathrm{U} / 1$ ) with normal CKMB isoenzyme. Pulmonary rales were heard on clinical cardiological examination. ECG showed sinus rhythm, negative T waves, missing $\mathrm{R}$ progression, and signs of left ventricular hypertrophy. Echocardiography showed left ventricular dilatation with severely reduced left ventricular function and abnormal left ventricular trabeculations in the apex. Abnormal left ventricular trabeculations were confirmed by cardiac magnetic resonance imaging. Angiography showed normal coronary arteries but left ventriculography showed abnormal structures protruding from the posterolateral wall and moving synchronously with the cardiac cycle (fig 1).

Clinical neurological examination showed diffuse, symmetrical limb wasting without weakness. Electromyography of the right brachial biceps muscle showed abundant fibrillations, fasciculations, and serial discharges but preserved motor unit architecture with a normal mean motor unit action potential duration. Serum lactate was normal at rest but increased at a workload of $30 \mathrm{~W}$ on a bicycle

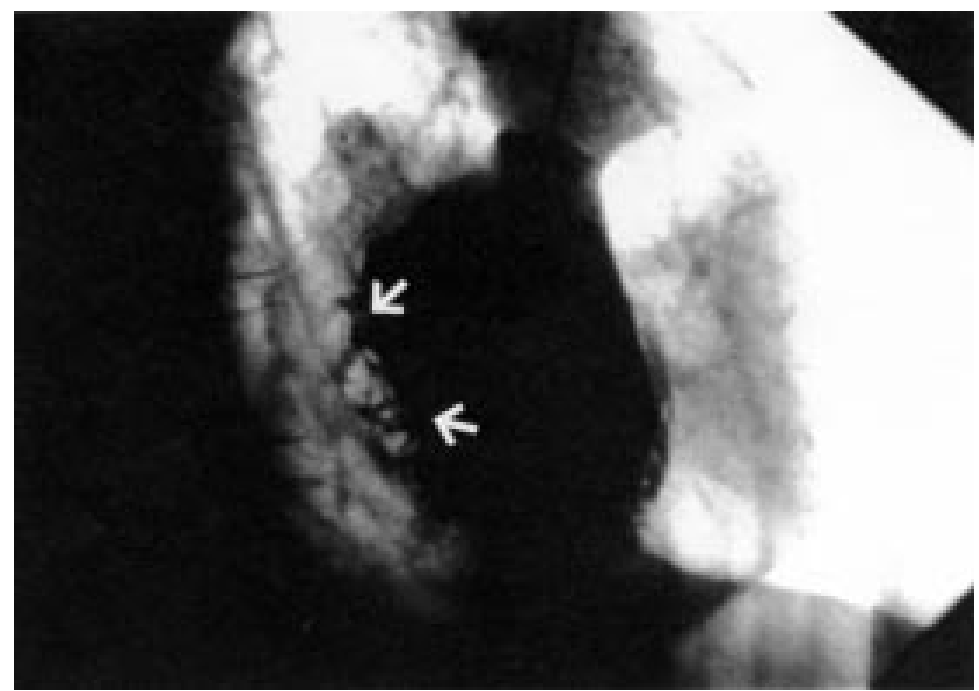

Figure 1 Left ventriculography in a $60^{\circ}$ left anterior oblique projection of a patient with mitochondriopathy. Abnormal structures protrude from the posterolateral wall; these were interpreted as abnormal left ventricular trabeculations. ergometer. ${ }^{2}$ Biopsy of the right lateral vastus muscle showed abnormalities typical of mitochondrial myopathy-like subsarcolemmal accumulation of mitochondria, reduced cytochrome $\mathrm{C}$ oxidase activity, and abnormally structured small mitochondria with proliferation of the mitochondrial cristae. ${ }^{3}$ Mitochondrial DNA analysis failed to detect any known or new mutation. At present the patient is doing well on ACE inhibitors and diuretics.

\section{Discussion}

Left ventricular trabeculations are structures that have the same echogenicity as the myocardium and move synchronously with it. They have to be distinguished from thrombi, false tendons, aberrant bands, and tumours according to established criteria. Left ventricular trabeculations can be normal or abnormal. Abnormal left ventricular trabeculations are defined as more than three prominent trabeculations apically to the papillary muscles. They are a rare cardiological finding of unknown aetiology and occur with or without congenital cardiac malformations. ${ }^{4}$ They may be associated with heart failure, left ventricular hypertrophy, and ECG abnormalities. Abnormal left ventricular trabeculations may be a subtype of hypertrophic cardiomyopathy. An argument that favours this hypothesis is that hypertrophic cardiomyopathy can be caused by mitochondriopathy. An argument for an association between abnormal left ventricular trabeculations and mitochondriopathy is the often occurring involvement of the heart in patients with mitochondriopathy. Two thirds of these patients have ECG and echocardiographic abnormalities. ${ }^{5}$ The clinical implication of our case is that patients in whom the diagnosis of mitochondriopathy is established should undergo a detailed cardiological examination.

In conclusion, abnormal left ventricular trabeculations may be a cardiac manifestation of mitochondrial myopathy.

1 Stöllberger C, Finsterer J, Blazek G, et al. Left ventricular non-compaction in a patient with Becker's muscular dystrophy. Heart 1996;76:380.

2 Finsterer J, Shorny S, Capek J, et al. Lactate stress test in the diagnosis of mitochondrial myopathy. F Neurol Sci 1998; 159:176-80.

3 Morgan-Hughes JA. The mitochondrial myopathies. In: Engel AG, Franzini-Armstrong C, eds. Myology. Basic and
clinical. New York: McGraw-Hill, 1994:1610-51.

4 Chin TK, Perloff JK, Williams RG, et al. Isolated noncompaction of left ventricular myocardium. A study of eight cases. Circulation 1990;82:507-13.

5 Finsterer J, Stöllberger C, Keller H, et al. Cardiac involvement in patients with myotonic dystrophy, Becker's muscular dystrophy and mitochondrial myopathy. Herz 1997;22:96-103. 